\title{
Strain and microstructures of GaN epilayers with thick InGaN interlayer grown by MOCVD
}

Jianxun Liu, Hongwei Liang*, Yang Liu, Xiaochuan Xia, Huolin Huang, Pengcheng Tao, Qasim Abbas Sandhu, Rensheng Shen, Yingmin Luo, Guotong Du

School of Physics and Optoelectronic Engineering, Dalian University of Technology, Dalian 116024, People's Republic of China

\section{Abstract}

GaN epilayers with thick InGaN interlayer are grown by metal-organic chemical vapor deposition on sapphire substrates. The as-grown GaN films with an InGaN interlayer show remarkable relaxed compressive strain measured by Raman spectroscopy. The microstructures within the InGaN interlayer were investigated by high resolution transmission electron microscopy. It indicated that the misfit dislocations and stacking faults in the InGaN interlayer formed, which is responsible for the relaxation of the lattice strain. In addition, the InGaN interlayer was found to terminate most of threading dislocation from the $\mathrm{GaN}$ pseudosubstrate layer even though the strain relaxation occurs. Such data help to provide further insight into the strain relaxation mechanisms and improve the quality of $\mathrm{GaN}$ films and the performance of $\mathrm{GaN}$ related devices.

Key words: GaN; InGaN interlayer; Strain; Microstructure;

\footnotetext{
${ }^{*}$ Corresponding author. E-mail: hwliang@dlut.edu.cn
} 


\section{Introduction}

GaN and related group-III nitride compounds are recognized to be excellent materials for applications in optoelectronics and high-power, high-temperature electronics [1-4]. However, high densities of threading dislocations (TDs, $\sim 10^{8}-10^{10} \mathrm{~cm}^{-2}$ ) have been reported to persist in $\mathrm{GaN}$ films due to the strain relaxation of hetero-epitaxial growth $[5,6]$. Dislocations are generally considered to be the leakage pathways and non-radiative recombination centers in $\mathrm{GaN}$ films, causing serious degradation of the device performance [7-9]. Thus, strain is an important issue for group-III nitride growth and strain engineering is essential to achieve high-quality $\mathrm{GaN}$ films. To date, various methods have been employed to the strain engineering to reduce the residual stress in the film. For examples, Reiher et al. [10] utilized a low temperature AlN interlayer to reduce the tensile stress and cracks in thick GaN layers on Si. Xu et al. [11] obtained a nearly stress-free GaN single-crystalline film with mirror-like morphology by inserting an InGaN interlayer as a weakly bonded layer. Dai et al. [12] introduced a novel porous substrate as a release layer to achieve high quality GaN crystals. Ma et al. [13] have reported the AlN/GaN superlattice interlayer for both stress and dislocation engineering, thus obtaining an enhanced optical output power of unpackaged light-emitting diode chips on Si substrates. However, previous literatures mainly focus on the final strain relaxation but there are only few reports on the detailed generation and propagation of the TDs after the introduction of various interlayers, as well as the micro-structural defects within the interlayer [14-16]. In our previous publication on unintentionally doped high resistivity GaN grown with an 
in-situ annealed InGaN interlayer, the interlayer-induced increase of the edge-type TDs was demonstrated by transmission electron microscopy (TEM) and high-resolution X-ray diffraction (HR-XRD) to reveal the high resistivity mechanisms [17]. However, considerable attention has been focused on the role of the edge-type TDs in determining the electrical properties of GaN epilayer. Moreover, the in-situ annealed InGaN interlayer show such significant degradation that few fine defect features can be characterized in detail to confirm which type of defect they are, and no strain relaxation mechanisms within the interlayer have been proposed in the literature. Deeply and extensively investigating the extended defects within/above the interlayer is favorable for revealing strain relaxation mechanisms and improving the quality of $\mathrm{GaN}$ films and the performance of $\mathrm{GaN}$ related devices.

Hence, in this report, the strain and micro-structural defects induced by the 60-nm-thick InGaN interlayer were studied in detail to reveal the strain relaxation mechanisms of GaN films. Cross-sectional TEM was used to identify the micro-structural defects as well as the generation and propagation of the TDs at the GaN/InGaN interface using two-beam diffraction conditions. Moreover, the residual stress in the as-grown films was also characterized by micro-Raman spectroscopy.

\section{Experimental details}

All samples were grown by an Aixtron $3 \times 2$ inch close-coupled showerhead MOCVD system on $c$-plane sapphire substrates. Trimethylgallium, trimethylindium and ammonia were used as $\mathrm{Ga}$, In and $\mathrm{N}$ sources, respectively. Triethylgallium was 
used as the Ga source for the growth of the InGaN. The schematic diagrams of the two samples structure can be found in Fig. 1(a) and Fig. 1(b), with the corresponding atomic force microscope (AFM) images and root mean square (RMS) roughness presented. For the growth of sample A, the sapphire substrate was firstly ramped to $1150{ }^{\circ} \mathrm{C}$ in hydrogen atmosphere to remove the surface damage and contamination induced by mechanical polish and preparation process. Then a thin low-temperature GaN buffer layer $(25 \mathrm{~nm})$ was grown on $c$-plane sapphire substrate at $530{ }^{\circ} \mathrm{C}$. Subsequently, a 3.2- $\mu$ m-thick unintentionally doped (UID) GaN film were deposited at $1050{ }^{\circ} \mathrm{C}$ followed by a 60 -nm-thick $\mathrm{InGaN}$ interlayer grown at $770{ }^{\circ} \mathrm{C}$. Finally, another 650-nm-thick UID GaN were grown on the InGaN interlayer at $1050{ }^{\circ} \mathrm{C}$. Sample B was a 4- $\mu$ m-thick UID GaN used as a reference to show the changes due to the incorporation InGaN interlayer.

The surface morphology of the samples was studied by Veeco D3100 AFM system, operated in tapping mode. The crystal quality and surface morphology of the GaN epilayers were investigated by HR-XRD. Residual stress in the epilayers was assessed by micro-Raman spectrometer in the backscattering geometry. Cross-sectional TEM were performed to characterize the microstructural defects induced by the InGaN interlayer in detail.

\section{Results and discussions}

The schematic diagrams and corresponding AFM images $\left(2.0 \times 2.0 \mu \mathrm{m}^{2}\right)$ for samples A and B are shown in Fig. 1(a) and (b), respectively. The GaN film with a 
thick InGaN interlayer, i.e., sample-A, exhibits a smooth surface morphology with the RMS roughness of $0.353 \mathrm{~nm}$. In contrast, sample-B reveals uniform atomic growth steps almost aligned parallel to the $c$ plane of $\mathrm{Al}_{2} \mathrm{O}_{3}$ with a RMS roughness of 0.282 nm. Several surface pits can be observed from each image, which may be associated with the terminations of threading dislocations [18]. Furthermore, the surface-steps can be observed clearly in the AMF images in Fig. 1, suggesting that both samples are dominated by a typical two-dimensional step-flow growth mode, which ensures a smooth and flat surface [19].
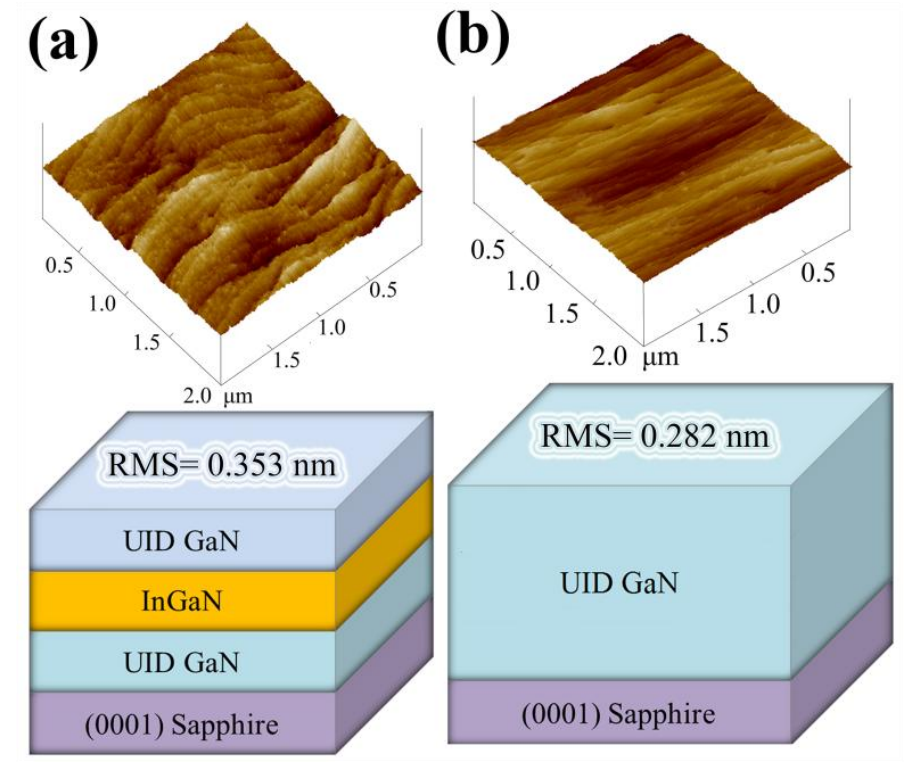

Fig. 1 Schematic diagrams and corresponding AFM images for (a) sample-A and (b) sample-B respectively. The RMS roughness from each sample is labeled.

The crystal quality of the two samples was characterized by HR-XRD $\omega$-scans using symmetrical (002) and asymmetrical (102) reflections. The FWHM of the (002) peak is 316 arc sec for sample A and 284 arc sec for sample B, corresponding to 
screw-type TDs (i.e., TDs with c-component Burgers vectors ) density of $1.0 \times 10^{8}$ $\mathrm{cm}^{-2}$ and $0.8 \times 10^{8} \mathrm{~cm}^{-2}$, respectively. Meanwhile, the FWHM values of the (102) diffraction peak are 438 and 325 arc sec for samples A and B respectively, which correspond to edge-type TDs (i.e., TDs with a-component Burgers vectors) density of $4.9 \times 10^{8} \mathrm{~cm}^{-2}$ and $2.7 \times 10^{8} \mathrm{~cm}^{-2}$, respectively. Details on the calculation of screwand edge-type TDs density can be found in our previous report [17]. Obviously, the total TDs density $D_{d i s}$ for sample-A $\left(5.9 \times 10^{8} \mathrm{~cm}^{-2}\right)$ is dramatically increased compared with that of sample-B $\left(3.5 \times 10^{8} \mathrm{~cm}^{-2}\right)$ and two reasons account for this. One is that the hetero-epitaxial $\mathrm{GaN}$ on defective InGaN pseudo-substrate layer naturally cause the degradation of crystal quality. The other is that the strain relaxation may occur at the InGaN/GaN interface to relax the corresponding stress, and therefore generate edge-type misfit dislocation near the interface which may bend upwards as a-type or (a + c)-type TDs [20].

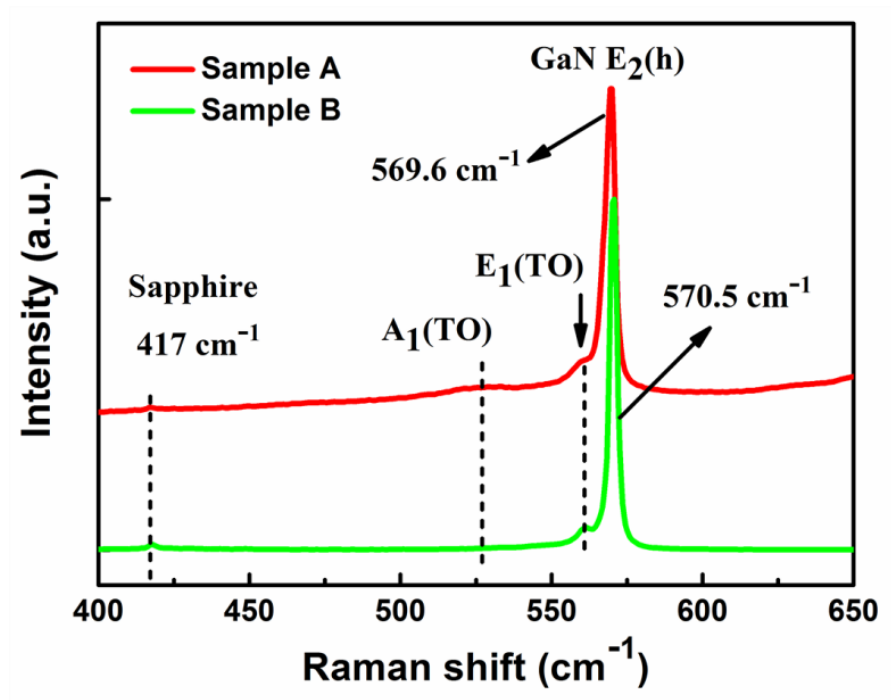

Fig. 2 Micro-Raman spectra from GaN epilayer with (red line)/without (green line) an InGaN interlayer grown on $c$-plane (0001) sapphire subtrates. 
Raman scattering has been extensively used to study III-nitrides and is a proven method for studying stress within the GaN epilayer [21]. Fig. 2 shows the Raman spectra of the two samples. The small peak at $417 \mathrm{~cm}^{-1}$ resulting from the sapphire phonon mode is usually used as a reference [6]. It is apparent that the $E_{2}$ (high) phonon frequency for samples A and B are blue-shifted with respect to the value of $568 \mathrm{~cm}^{-1}$ for bulk GaN, implying the presence of in-plane compressive strain in these GaN epilayers. Moreover, compared with the $\mathrm{E}_{2}$ (high) phonon frequency of sample $\mathrm{B}$ $\left(570.5 \mathrm{~cm}^{-1}\right)$, the $\mathrm{E}_{2}$ (high) phonon frequency for sample $\mathrm{A}\left(569.6 \mathrm{~cm}^{-1}\right)$ shows an obvious redshift of $0.9 \mathrm{~cm}^{-1}$, indicating that strain was partially relaxed when an InGaN interlayer was introduced. In order to evaluate the changes in residual stress caused by the InGaN interlayer, a typical formula was utilized [22]:

$$
\Delta \boldsymbol{\omega}=K \cdot \boldsymbol{\sigma}
$$

where the $\sigma(\mathrm{GPa})$ is the residual stress, $\Delta \omega\left(\mathrm{cm}^{-1}\right)$ is the Raman frequency shift of a given phonon mode, $K$ is the linear stress coefficient. For GaN epilayers grown on sapphire, the value of stress coefficient $K$ for the $\mathrm{E}_{2}$ (high) phonon is $2.56 \mathrm{~cm}^{-1} \mathrm{GPa}^{-1}$. With the data above, it can be calculated that the residual compressive stress in samples A and B are $0.82 \mathrm{GPa}$ and $1.17 \mathrm{GPa}$, respectively. The results indicate that the residual stress in sample $\mathrm{A}$ was reduced by $0.35 \mathrm{GPa}$, yet reveals the InGaN interlayer's tailoring ability to reduce the residual stress in GaN epilayer. In addition, two unsolved peaks near the principal $\mathrm{E}_{2}$ (high) peaks, i.e., $\mathrm{A}_{1}(\mathrm{TO})$ and $\mathrm{E}_{1}(\mathrm{TO})$ peaks, are visible at 527 and $561 \mathrm{~cm}^{-1}$ respectively for sample $A$ and only $E_{1}(\mathrm{TO})$ appears for sample B. The appearance of these peaks is still a subject under debate and is 
generally attributed to pyramidal growth of the GaN before coalescence [23].

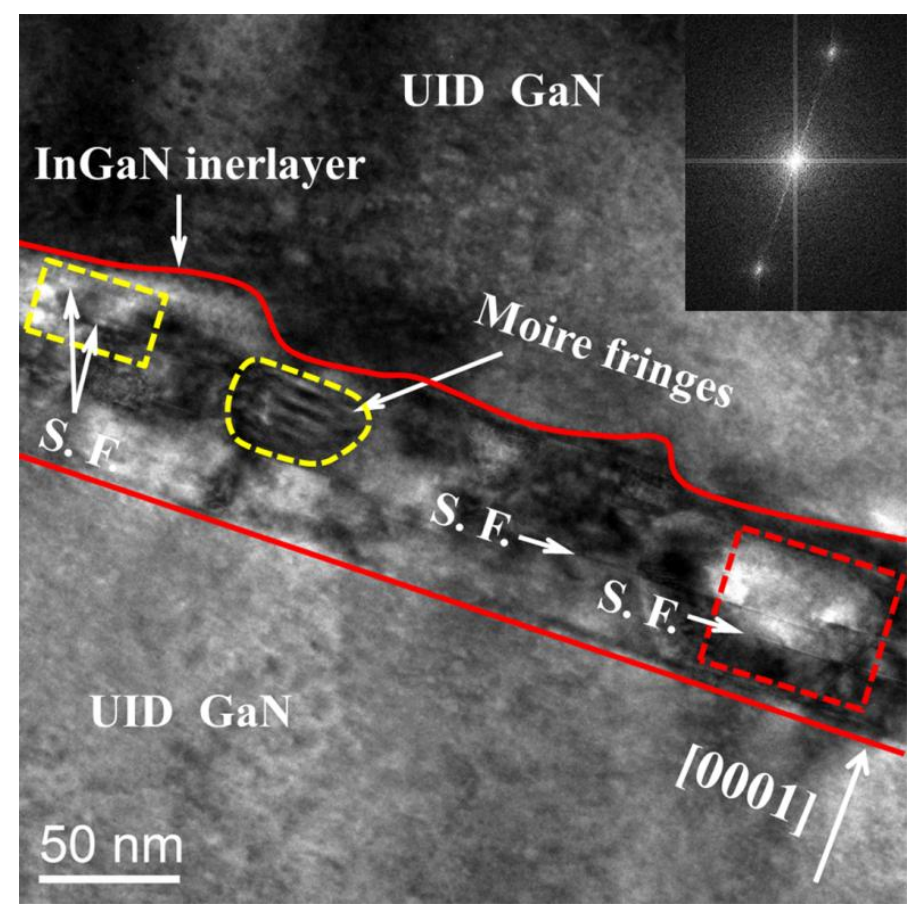

Fig. 3 Cross-sectional bright-field TEM micrograph of sample-A taken near the [11 $\overline{2} 0]$ zone axis. The top inset is the corresponding FFT pattern of the red square-marked area.

To investigate the micro-structural defects induced by the InGaN interlayer, a cross-sectional bright-field TEM micrograph was taken near the [11 $\overline{2} 0]$ zone axis to reveal the overview of the microstructures within the InGaN interlayer, as shown in Fig. 3. Under this imaging condition, the image is sensitive to atomic mass density. Moire fringes can be found within the InGaN interlayer (marked by yellow circle in Fig. 3), which is attributed to the difference between the lattice constants of the In-rich InGaN (dark contrast) and the surrounding matrix [24]. The as-grown sample demonstrates quite diffusive distribution of indium and the rough positions of the InGaN interlayer can be identified which were outlined by the red lines. The lower 
InGaN/GaN interface appears to be abrupt, while the upper interface shows roughness, which may be attributed to the compositional pulling effects in InGaN layer. In addition, stacking faults (S.F.) can be observed clearly within the $\mathrm{InGaN}$ interlayer, which was reported to be caused by the stacking order mismatch between the columnar grains in highly strained InGaN layer [25]. Nonuniform contrast and large amounts of S.F defects indicate significant phase separation and deteriorated crystal quality of the InGaN interlayer. To further verify this, a selective area fast Fourier transform (FFT) of the red square-marked area was also shown as the top inset in Fig. 3. The splitting of the diffraction pattern along $\mathrm{c}$ axis proved that strain relaxation occurred indeed within the InGaN layer [26].

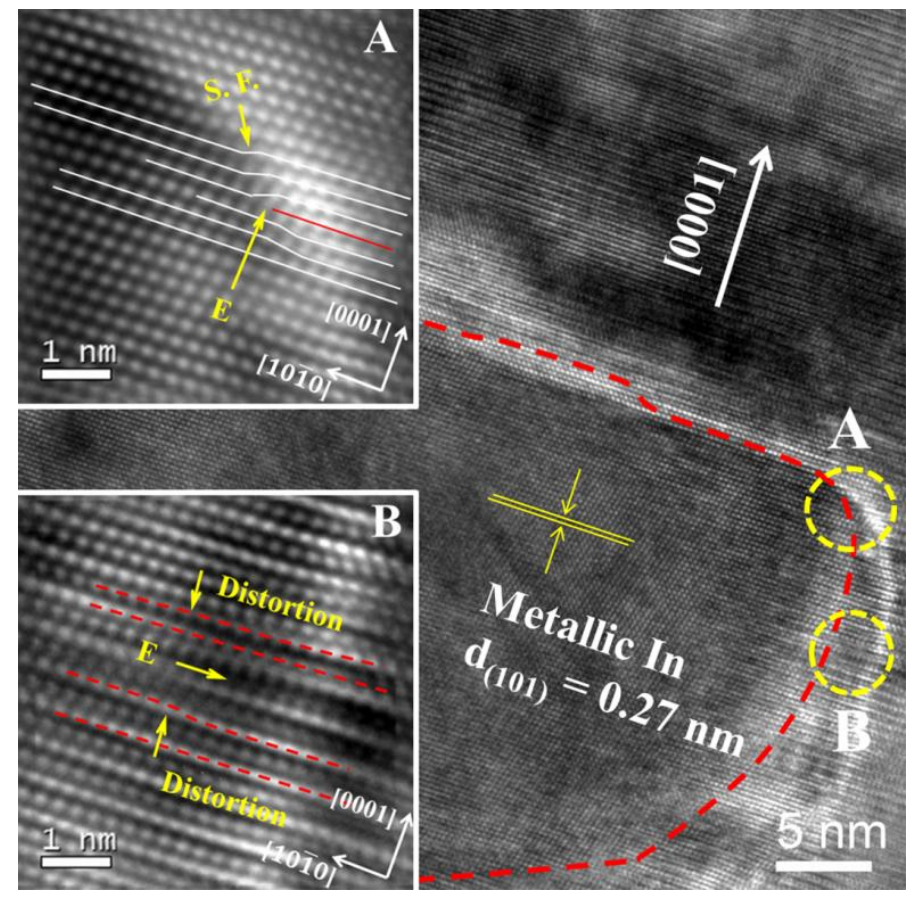

Fig. 4 High-resolution TEM image of the In-rich region marked by the yellow-dashed circle in Fig. 3; The top and bottom insets are the corresponding IFFT images of the circle-marked zone-A and zone-B. 
To visualize directly the stacking faults and local distortions of atomic arrangement in the InGaN layer, high-resolution TEM was carried out as shown in Fig. 4. The red dashed lines outline a region of In-rich precipitate and the In/Ga interface is relatively sharp. By measuring the $d$-spacing along GaN [0001] orientation, the In-rich precipitate was determined to be pure metallic In $\left(d_{(101)}=0.27 \mathrm{~nm}\right)$ which has a fixed orientation relation with the GaN matrix: $(101)_{\mathrm{In}} \|(0001)_{\mathrm{GaN}}$. The top inset-A and bottom inset-B in Fig. 4 are corresponding atomic-resolved inversed fast Fourier transform (IFFT) image of the metallic In/InGaN interface marked with "A" and "B", respectively. Clear stacking faults (marked with "S.F.") and edge misfit dislocations (marked with "E") can be observed in the inset-A and local distortions of atomic arrangement of the $\mathrm{In} / \mathrm{InGaN}$ interface can be found in the inset-B. By measuring the strained lattice constants (i.e., c lattice parameter along $z$ direction) at different depth of the InGaN layer, the out-of-plane strain $\varepsilon_{z z}$ can be given according to the relation $[27,28]:$

$$
\varepsilon_{\mathbf{z Z}}=\frac{c_{0}^{G a N}-c_{I n G a N}}{c_{\text {InGaN }}}
$$

where $c_{0}^{G a N}$ and $c_{I n G a N}$ are the unstrained lattice constants of $\mathrm{GaN}\left(c_{0}^{G a N}=0.5185 \mathrm{~nm}\right)$ and strained InGaN measured by HR-TEM, respectively. Hence, the lattice strain $\left(\varepsilon_{z z}\right)$ obtained from Eq. (4) versus thickness of InGaN layer starting from the bottom InGaN/GaN interface is shown in Fig. 5. The residual strain initially increases within 10-nm thickness but swiftly drops to minimum at around $20 \mathrm{~nm}$. This reduction of the lattice strain can be attributed to the partial relaxation of the InGaN layer at the critical thickness [29]. Therefore, the critical thickness for InGaN interlayer can be 
limited at the range of $10-20 \mathrm{~nm}$ in the present samples. Afterwards, the lattice strain continuously increases over depth, which can be well accounted for by the linear increase of In content, i.e., "the composition pulling effects" [30] or "up-hill" diffusion of indium atoms [31]. These observations quantitatively confirm the critical thickness of the InGaN interlayer (although not exact numbers) and visualize the species of the microstructure defects in detail, which can serve as a reference for the analysis of the strain relaxation of InGaN.

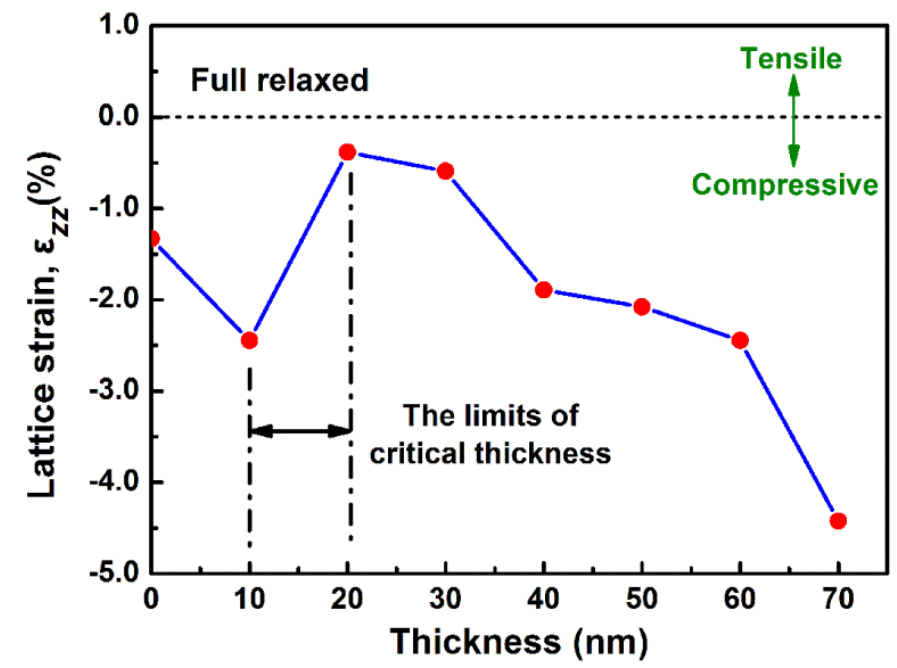

Fig. 5 The lattice strain $\left(\varepsilon_{z z}\right)$ obtained from Eq. (4) versus thickness of the InGaN interlayer.

The dotted line indicates the fully relaxed InGaN. 


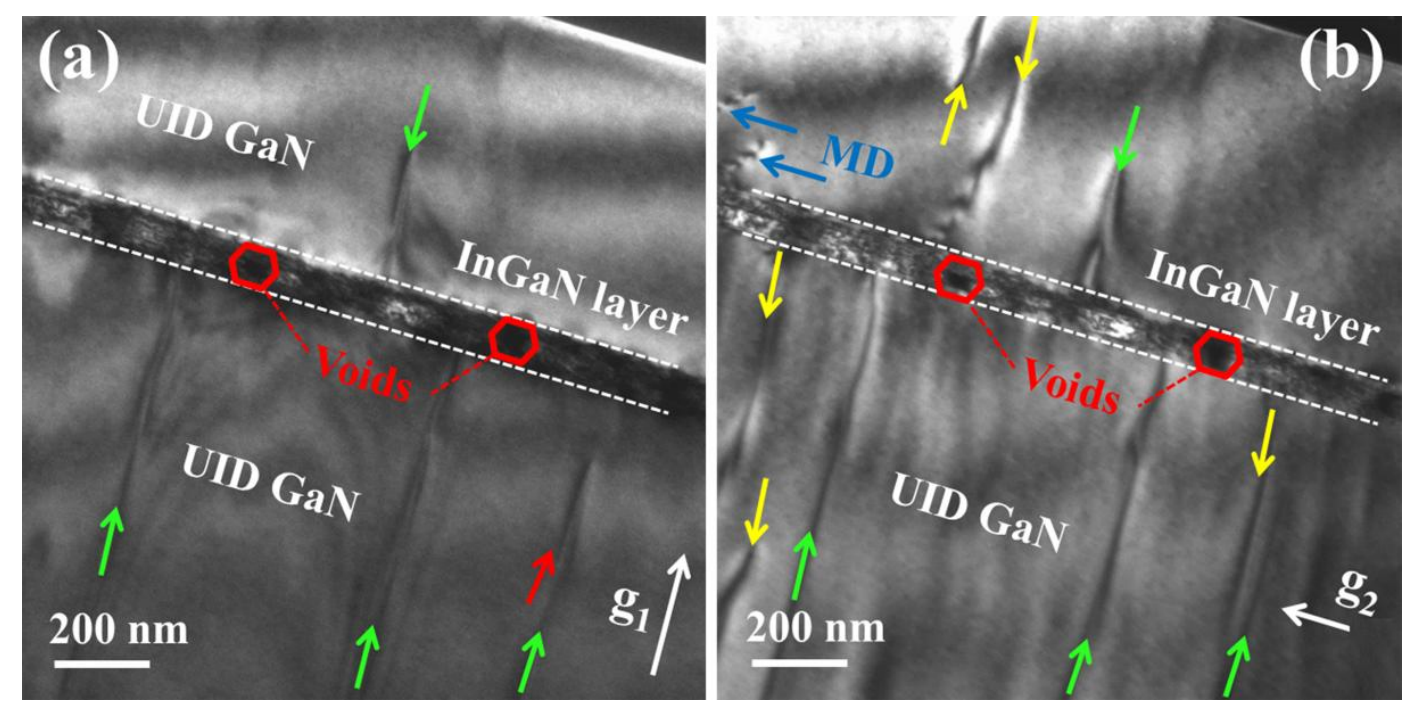

Fig. 6 Cross-sectional TEM images for sample-A showing a detailed view of the dislocations with (a) $g_{1}=[0002]$ and (b) $g_{2}=[11 \overline{2} 0]$ imaging conditions. Yellow, red, and green arrows indicate $\boldsymbol{a}, \boldsymbol{c}$ and $(\boldsymbol{a}+\boldsymbol{c})$ type TDs, respectively.

Figs. 6(a) and 6(b) show representative cross-sectional TEM images taken from the same area under different diffracting conditions. According to $\mathbf{g} \cdot \mathbf{b}$ dislocation invisibility criterion [32], all types of dislocations, i.e., $\boldsymbol{a}$-, $\boldsymbol{c}$ - and $(\boldsymbol{a}+\boldsymbol{c})$-type TDs, can be identified in the films and here they were indicated by yellow, red and green arrows, respectively. It is worthy to note that all of the observed TDs beneath the InGaN interlayer stop propagating upward, indicating that the penetration of TDs was completely blocked by the InGaN interlayer. Nevertheless, $\boldsymbol{a}$-type misfit dislocations (marked with "MD" and blue arrows in Fig. 6) and new TDs with an $\boldsymbol{a}$ component appear above the interface. These subsequent dislocations may be related to strain relaxation at the InGaN/GaN interface. In spite of this, the GaN film above the InGaN interlayer show reduced TDs density compared with that of the GaN underlying layer by counting the dislocation lines in Fig. 6. Voids and inhomogeneous diffraction 
contrast can also be found in the InGaN interlayer, suggesting that there is a significant degradation of the structural properties.

\section{Conclusions}

In summary, the strain and microstructures of GaN epilayers with thick InGaN interlayer have been investigated. Particular microstructures such as TDs, MDs, S.F. and metallic In precipitates are visualized in detail. Compressive strain of the GaN epilayer was dramatically relaxed by introducing a thick InGaN interlayer. The thick InGaN interlayer was found to relax the intrinsic compressive strain at the critical thickness by the formation of MDs and S.F.. In addition, the InGaN interlayer was found to terminate most of the TDs from the GaN pseudosubstrate layer if well designed to avoid new MDs and edge-type TDs generation. The research in this paper helps us to better understand the strain relaxation mechanisms and improve the quality of GaN films and the performance of $\mathrm{GaN}$ related devices. 


\section{Acknowledgements}

This work was supported by National Key R\&D Plan (Nos.2016YFB0400600, 2016YFB0400601), National Science Foundation of China (Nos. 11675198, 61376046, 11405017, 61574026), the Fundamental Research Funds for the Central Universities (Nos. DUT15LK15, DUT15RC(3)016, No.DUT16LK29), Liaoning

Provincial Natural Science Foundation of China (Nos.2014020004, 201602453), China Postdoctoral Science Foundation Funded Project (No.2016M591434), Jiangxi Provincial Natural Science Foundation of China (No.20133ACB20005), The Open

Fund of the State Key Laboratory on Integrated Optoelectronics (No.IOSKL2015KF18, No.IOSKL2015KF22). 


\section{Reference}

[1] Nakamura S. Background Story of the Invention of Efficient InGaN Blue-Light-Emitting Diodes (Nobel Lecture). Angew Chem Int Ed. 2015;54:7770-88.

[2] Sang L, Liao M, Ikeda N, Koide Y, Sumiya M. Enhanced performance of InGaN solar cell by using a super-thin AlN interlayer. Appl Phys Lett. 2011;99:161109.

[3] Li D, Sun X, Song H, Li Z, Chen Y, Jiang H, et al. Realization of a High - Performance GaN UV Detector by Nanoplasmonic Enhancement. Adv Mater. 2012;24:845-9.

[4] Huang H, Liang YC, Samudra GS, Chang T-F, Huang C-F. Effects of gate field plates on the surface state related current collapse in AlGaN/GaN HEMTs. IEEE Transactions on Power Electronics. 2014;29:2164-73.

[5] Hino T, Tomiya S, Miyajima T, Yanashima K, Hashimoto S, Ikeda M. Characterization of threading dislocations in GaN epitaxial layers. Appl Phys Lett. 2000;76:3421-3.

[6] Liu X, Li D, Sun X, Li Z, Song H, Jiang H, et al. Stress-induced in situ epitaxial lateral overgrowth of high-quality GaN. Crystengcomm. 2014;16:8058-63.

[7] Cherns D, Henley S, Ponce F. Edge and screw dislocations as nonradiative centers in InGaN/GaN quantum well luminescence. Appl Phys Lett. 2001;78:2691-3.

[8] Young NG, Farrell RM, Hu YL, Terao Y, Iza M, Keller S, et al. High performance thin quantum barrier InGaN/GaN solar cells on sapphire and bulk (0001) GaN substrates. Appl Phys Lett. 2013;103:173903.

[9] Fireman MN, Browne DA, Mazumder B, Speck JS, Mishra UK. Demonstration of isotype GaN/AlN/GaN heterobarrier diodes by NH3-molecular beam epitaxy. Appl Phys Lett. 2015;106:202106.

[10] Reiher A, Bläsing J, Dadgar A, Diez A, Krost A. Efficient stress relief in GaN heteroepitaxy on Si (111) using low-temperature AlN interlayers. J Cryst Growth. 2003;248:563-7.

[11] Xu X, Guo Y, Liu X, Liu J, Song H, Zhang B, et al. GaN grown with InGaN as a weakly bonded layer. CrystEngComm. 2011;13:1580-5.

[12] Dai Y, Wu Y, Zhang L, Shao Y, Tian Y, Huo Q, et al. A novel porous substrate for the growth of high quality GaN crystals by HVPE. RSC Adv. 2014;4:35106-11.

[13] Ma J, Zhu X, Wong KM, Zou X, Lau KM. Improved GaN-based LED grown on silicon (111) substrates using stress/dislocation-engineered interlayers. J Cryst Growth. 2013;370:265-8.

[14] Jiang H, Egawa T, Hao M, Liu Y. Reduction of threading dislocations in AlGaN layers grown on AlN/sapphire templates using high-temperature GaN interlayer. Appl Phys Lett. 2005;87:241911.

[15] Li L, Yang La, Cao R, Xu SR, Zhou X, Xue J, et al. Reduction of threading dislocations in N-polar GaN using a pseudomorphicaly grown graded-Al-fraction AlGaN interlayer. J Cryst Growth. 2014;387:1-5.

[16] Wang T-Y, Ou S-L, Horng R-H, Wuu D-S. Improved GaN-on-Si epitaxial quality by incorporating various SixNy interlayer structures. J Cryst Growth. 2014;399:27-32.

[17] Liu JX, Liang HW, Li BH, Liu Y, Xia XC, Huang HL, et al. Unintentionally doped high resistivity GaN layers with an InGaN interlayer grown by MOCVD. RSC Adv. 2016;6:60068-73.

[18] Wu X, Brown L, Kapolnek D, Keller S, Keller B, DenBaars S, et al. Defect structure of metal organic chemical vapor deposition - grown epitaxial (0001) GaN/Al2O3. J Appl Phys. 1996;80:3228-37. 
[19] Ting SM, Ramer JC, Florescu DI, Merai VN, Albert BE, Parekh A, et al. Morphological evolution of InGaN/GaN quantum-well heterostructures grown by metalorganic chemical vapor deposition. J Appl Phys. 2003;94:1461-7.

[20] Lotsari A, Kehagias T, Tsiakatouras G, Tsagaraki K, Katsikini M, Arvanitidis J, et al. Structural anisotropic properties of a-plane GaN epilayers grown on r-plane sapphire by molecular beam epitaxy. J Appl Phys. 2014;115:213506.

[21] Zhang HD, Shao YL, Zhang L, Hao XP, Wu YZ, Liu XY, et al. Growth of high quality GaN on a novel designed bonding-thinned template by HVPE. Crystengcomm. 2012;14:4777-80.

[22] Zhao DG, Xu SJ, Xie MH, Tong SY, Yang H. Stress and its effect on optical properties of GaN epilayers grown on $\mathrm{Si}(111)$, 6H-SiC(0001), and c-plane sapphire. Appl Phys Lett. 2003;83:677-9.

[23] Pophristic M, Long FH, Schurman M, Ramer J, Ferguson IT. Raman microscopy of lateral epitaxial overgrowth of GaN on sapphire. Appl Phys Lett. 1999;74:3519-21.

[24] McCluskey MD, Romano LT, Krusor BS, Bour DP, Johnson NM, Brennan S. Phase separation in InGaN/GaN multiple quantum wells. Appl Phys Lett. 1998;72:1730-2.

[25] Wu X, Kapolnek D, Tarsa E, Heying B, Keller S, Keller B, et al. Nucleation layer evolution in metal - organic chemical vapor deposition grown GaN. Appl Phys Lett. 1996;68:1371-3.

[26] Qian F, Li Y, Gradecak S, Park HG, Dong YJ, Ding Y, et al. Multi-quantum-well nanowire heterostructures for wavelength-controlled lasers. Nature Materials. 2008;7:701-6.

[27] Holec D, Costa PMFJ, Kappers MJ, Humphreys CJ. Critical thickness calculations for InGaN/GaN. J Cryst Growth. 2007;303:314-7.

[28] Jana SK, Mukhopadhyay P, Ghosh S, Kabi S, Bag A, Kumar R, et al. High-resolution X-ray diffraction analysis of AlxGa1-xN/InxGa1-xN/GaN on sapphire multilayer structures: Theoretical, simulations, and experimental observations. J Appl Phys. 2014;115.

[29] Park I-K, Kwon M-K, Baek S-H, Ok Y-W, Seong T-Y, Park S-J, et al. Enhancement of phase separation in the InGaN layer for self-assembled In-rich quantum dots. Appl Phys Lett. 2005;87:061906.

[30] Pereira S, Correia MR, Pereira E, O'Donnell KP, Trager-Cowan C, Sweeney F, et al. Compositional pulling effects inInxGa1-xN/GaNlayers: A combined depth-resolved cathodoluminescence and Rutherford backscattering/channeling study. Phys Rev B. 2001;64.

[31] Feng S-W, Tang T-Y, Lu Y-C, Liu S-J, Lin E-C, Yang CC, et al. Cluster size and composition variations in yellow and red light-emitting InGaN thin films upon thermal annealing. J Appl Phys. 2004;95:5388-96.

[32] Wu F, Tyagi A, Young E, Romanov A, Fujito K, DenBaars S, et al. Misfit dislocation formation at heterointerfaces in $(\mathrm{Al}, \mathrm{In}) \mathrm{GaN}$ heteroepitaxial layers grown on semipolar free-standing $\mathrm{GaN}$ substrates. J Appl Phys. 2011;109:033505. 